\title{
Early Provisional Estimates of Drug Overdose, Suicide, and Transportation-related Deaths: Nowcasting Methods to Account for Reporting Lags
}

\author{
Lauren M. Rossen, Ph.D., Division of Research and Methodology; \\ Holly Hedegaard, M.D., Division of Analysis and Epidemiology; Margaret Warner, Ph.D., \\ Farida B. Ahmad, M.P.H., and Paul D. Sutton, Ph.D., Division of Vital Statistics, \\ National Center for Health Statistics
}

\section{Ahstract}

Objective-To describe nowcasting methods to generate provisional estimates of drug overdose, suicide, and transportation-related deaths.

Methods-Deaths due to drug overdose, suicide, and transportation were identified using International Classification of Diseases, 10th Revision underlying cause-of-death codes. Using a multi-stage hierarchical Bayesian modeling process, models were fit to predict weekly counts of deaths under different assumptions about how timeliness of provisional data has changed in 2020 relative to 2019. Model performance was evaluated by fitting models using provisional data from May 2020 and comparing the predicted counts with the reported provisional counts that became available 6-11 months later. Hierarchical Bayesian models were then fit to estimate the trends in deaths due to the three causes from early 2016 through October 24, 2020.

Results - On average, predicted counts assuming jurisdiction-specific improvements in timeliness since 2019 were within $5 \%$ of the eventual reported counts for the United States through April 5, 2020. The accuracy of the predicted counts varied depending on assumptions about how timeliness has changed. Predicted weekly numbers of drug overdose deaths increased from early 2020 through May 2020, then plateaued or declined from June through late October. Predicted weekly numbers of suicide deaths were similar to historic levels in early 2020, then declined from March through June, and were similar to historic levels through late October. Predicted weekly numbers of transportation-related deaths were similar to historic levels in early 2020 , then declined from March through April, and subsequently increased.

Conclusion - The nowcasting models for the three causes of death used in this evaluation provided good agreement with observed estimates through early April 2020. These methods can be used to provide preliminary estimates for recent time periods, which are important to understand the impact of the Coronavirus Disease 2019 pandemic on injury-related causes of death.

Keywords: mortality surveillance • National Vital Statistics System • Vital Statistics Rapid Release • provisional data

\section{Introduction}

The National Center for Health Statistics (NCHS) uses mortality data from the National Vital Statistics System (NVSS) to monitor trends in death rates from various causes (1). Final mortality data are generally not available until 11-13 months following the close of the calendar year. In order to provide more timely information on trends in mortality, NCHS developed methods to generate provisional estimates of mortality for many causes of death $(2,3)$. These estimates are published quarterly or monthly through the Vital Statistics Rapid Release (VSRR) program $(2,3)$.

Provisional estimates are based on a snapshot of all the mortality data received and processed by NCHS as of a specified date. To ensure estimates are sufficiently complete (i.e., greater than $90 \%$ of death records are available for analysis), provisional mortality data are published with a 3- to 9-month lag from the time the deaths occurred.

Several reports have described the timeliness of provisional mortality data, and how timeliness varies by cause of death, age at death, and jurisdiction of occurrence (4-8). Generally, the timeliness of provisional mortality data is slower for external causes of death such as drug overdose and suicide, though timeliness has improved steadily in recent years for all deaths and for external-cause deaths. For example, in $2015,84 \%$ of all death records were available for analysis within 13 weeks of the date of death, but only $38 \%$ of drug overdose death records were available within 13 weeks (7). By 2017, 95\% of death records overall and $70 \%$ of drug overdose death records were available within 13 weeks of the date of death (4). 
Continued improvements in the timeliness of NVSS mortality data have enabled more timely surveillance of key mortality outcomes. In April 2020, NCHS began publishing provisional estimates of Coronavirus Disease 2019 (COVID-19) mortality (https://www.cdc. gov/nchs/nvss/covid-19.htm) (9). NCHS now publishes daily and weekly updates of counts of death due to COVID-19 and other causes of death to provide data that researchers can use to examine the impact of the pandemic on various mortality outcomes (9). External causes of death, including drug overdose and suicide, were not initially included in these provisional data releases because the data for these types of deaths are typically not sufficiently complete (i.e., fewer than $90 \%$ of death records are available for analysis) until 6 to 9 months after the date of death. Thus, while there is an urgent need for data on external causes of death, particularly to identify the impact of the pandemic, available provisional data are not complete enough to ensure accurate estimates of the number of deaths due to external causes in more recent months.

Nowcasting, or predicting the number of events that have occurred when a certain proportion has not yet been reported, has been used to account for reporting lags in disease surveillance (10-12) and can provide more current estimates of a given outcome even with incomplete data. Nowcasting methods, as applied to mortality data, rely on understanding and quantifying delays in reporting and accounting for those delays in models predicting the outcome of interest. While provisional data from past years can be used to estimate reporting delays, the timeliness of provisional mortality data has been improving in recent years, particularly for drug overdose deaths $(4,7)$. As a result, prior estimates of reporting lags may not be accurate for 2020 data. Consequently, it is important to account for potential improvements or changes in reporting lags when estimating provisional counts of deaths.
This report describes an approach for estimating trends in drug overdose, suicide, and transportation-related deaths from January 1, 2020, to October 24, 2020, using nowcasting methods to account for reporting lags. The first objective was to examine the accuracy of estimates under alternative scenarios where different assumptions were made about how timeliness may have changed in 2020 compared with 2019. The second objective was to generate estimates of trends in each of the outcomes and examine how deaths from these causes have changed in recent weeks relative to prior years.

\section{Methods}

\section{Data}

Aggregated weekly counts of deaths by cause and jurisdiction where the death occurred, from 2016 through the most recent week, were tabulated from NVSS provisional mortality data (i.e., data that were received, coded, and available for analysis as of November 22, 2020).

Deaths due to specific causes were identified using International Classification of Diseases, 10th Revision (ICD-10) underlying cause-of-death codes. Deaths due to drug overdose were identified using X40-X44, X60-X64, X85, and Y10-Y14; deaths due to suicide using codes $\mathrm{U} 03, \mathrm{X} 60-\mathrm{X} 84$, and Y87.0; and deaths due to transportation using V01-V99 and Y85. Of note, 94\% of transportation deaths were due to motor vehicle traffic deaths.

Data for deaths occurring in 52 vital statistics jurisdictions representing the 50 states, District of Columbia, and New York City were included. Data from Puerto Rico were excluded; data from the remaining four territories were not available for inclusion in provisional data releases.

\section{Analysis}

\section{Overview}

To estimate recent trends in drug overdose, suicide, and transportation-related deaths using available provisional data and accounting for reporting delays, a multi-stage hierarchical Bayesian modeling process was conducted (see the box on page 3). Briefly, in Stage 1, a set of models were fit to estimate how complete provisional data were for each outcome, by jurisdiction of occurrence and lag (length of time between when the death occurred and when the provisional data were available, in weeks), based on provisional data from 2018 through 2019. Predicted estimates of completeness from these models were used to create several sets of weights that could be applied to current provisional counts of deaths, to account for the incomplete reporting under different assumptions about how timeliness has changed since 2019. In Stage 2, these weights were applied to the most recent provisional data, and models were fit to estimate the trends in deaths due to the three causes from early 2016 through October 24, 2020. Finally, to validate the models and evaluate which assumptions about timeliness may be more realistic, models were fit using provisional data from May 10, 2020, for the time period including the weeks ending December 15, 2019, through April 5, 2020, and compared with the reported counts as of November 22, 2020. Detailed information about each stage and the validation process is provided below. 


\section{Stage 1: Develop weights to account for incomplete reporting of deaths}

- Model the completeness of weekly provisional data from 2018 through 2019 by:

- Outcome

- Jurisdiction

- Lag (weeks) after the deaths occurred

- Predict completeness (provisional count divided by final count) for each outcome, jurisdiction, and lag, under different assumptions about timeliness:

- No change in timeliness since 2019 (median level from 2019)

- Moderate improvement since 2019 (75th percentile)

- Extreme improvement since 2019 (95th percentile)

- Create weights for each jurisdiction and lag from predicted completeness values:

- Three outcomes (drug overdose, suicide, transportation)

- Three timeliness scenarios

\section{Stage 2: Model weekly trends in injury-related causes of death}

- For each of the three outcomes and three different timeliness scenarios, model the weighted number of deaths using the most recent provisional data

- Nine different models

- From each model, predict weekly number of deaths for each jurisdiction and 95\% Bayesian Credible Intervals (95\% BCI)

\section{Validation}

- For each of the three outcomes and three timeliness scenarios, fit Stage 2 models using provisional data available on May 10, 2020

- Nine different models

- From each model, predict the weekly number of deaths occurring the weeks ending December 15, 2019, through April 5, 2020

- Compare predicted values to reported values from the most recent provisional data (November 22, 2020)

- Percent difference between the model-based estimates and the observed counts

- Root mean squared error (RMSE)

- Trend plots

\section{Stage 1: Developing weights to account for incomplete reporting of deaths}

To determine how complete provisional data are at different lags (e.g., 1 week following the date of death, 2 weeks following), a set of hierarchical Bayesian models were used to estimate the completeness of provisional data. Completeness was defined as the provisional number of deaths divided by the final number of deaths. For example, if the final number of deaths occurring in the week ending January 7 , 2018, was 100 and the provisional number as of January 14, 2018, (1-week lag) was 20, completeness would be $20 \%$.

The final mortality data for 2018 were used to tabulate the counts of deaths by jurisdiction of occurrence and week of death. At the time of analysis, final 2019 data were not yet available, so provisional data from early July 2020 were used to approximate the final data for 2019. Typically, provisional data for a given data year are over $99 \%$ complete by the July following the close of the data year, when the data are finalized for the production of final data files and related reports. The 2019 final data was published in December 2020.

Completeness of weekly provisional data from 2018 through 2019 was modeled using hierarchical Bayesian zero-inflated binomial models with random effects for jurisdiction of occurrence, time (in weeks), lag (from 1 through 52 weeks), a time-by-jurisdiction interaction, and a lag-by-jurisdiction interaction. These latter two interaction terms allow for both temporal trends and for the lag in reporting to vary by jurisdiction. The time and lag random effects were specified using type I random walk distributions (where values for a given week depend on values from the prior week), which allow for nonlinear temporal patterns. The remaining random effects (jurisdiction, time-by-jurisdiction interaction, and lag-by-jurisdiction interaction) were specified as independent and identically distributed (IID). Spatially structured random effects (Besag, York, Mollié; BYM) for each jurisdiction were also considered (13), but model fit was not improved, based on the WatanabeAkaike Information Criterion (WAIC) (14).

For each outcome (i.e., drug overdose, suicide, transportation), separate models were fit to generate estimates of completeness of provisional data at various lags. Posterior predicted values of completeness (i.e., median, 75th percentile, and 95th percentile) were obtained from each of the models and used to generate sets of weights for each jurisdiction and lag (in weeks), where the weights were defined as the inverse of completeness. Three sets of weights were developed in order to account for potential changes in timeliness of provisional data since the beginning of 2020 (potentially related to the COVID-19 pandemic). Generally, timeliness of provisional data has been improving (5) and there is no indication that COVID-19 negatively impacted timeliness overall. Therefore, scenarios where timeliness is worse in 2020 than it was in 2019 were not explored. 
- The first set of weights was based on the median values of completeness for weekly 2019 provisional data. This set of weights assumes that timeliness in 2020 is similar to the median levels seen in 2019.

- The second set of weights uses the 75th percentile of estimated completeness for weekly 2019 provisional data. This set of weights assumes moderate improvements in timeliness, where completeness in 2020 is roughly equivalent to the 75 th percentile of completeness for weekly provisional data in 2019.

- The third set of weights relies on the 95th percentile of estimated completeness for weekly 2019 provisional data. This set of weights assumes extreme improvements in timeliness, where completeness in 2020 is equivalent to the 95 th percentile of completeness for weekly provisional data in 2019.

\section{Stage 2: Modeling weekly trends in injury-related causes of death}

For each outcome and set of weights, separate hierarchical Bayesian zeroinflated Poisson models were fit to generate estimates of weekly provisional counts of deaths for that outcome. These models were used to generate posterior predicted counts of deaths by jurisdiction, week, and cause of death under different assumptions about the timeliness of data in 2020. The models included a spatially structured random effect (BYM) for jurisdiction, a type I random walk for time (week of death), and an IID random effect for the timeby-jurisdiction interaction. These terms allow for the number of deaths in a given jurisdiction to depend on the values for the neighboring jurisdictions (based on Delaunay triangulation (15)) and for the temporal trends to vary by jurisdiction. Poisson models were also explored but did not result in an improvement in fit, based on the WAIC. See the Technical Notes for more detail about the models.

Weights for the most recent 4 weeks (i.e., lag of zero, corresponding to deaths reported in the same week they occurred, through a lag of 3 weeks) were highly unstable for all outcomes, and those weeks were excluded from analysis. Additionally, some jurisdictions had historically lower levels of completeness or very small numbers of deaths, which resulted in highly inflated predicted estimates. For example, Connecticut and North Carolina have historically relied on paper-based systems for death registration, and have had lower levels of completeness, especially at shorter lags. Weights were subsequently truncated to assume at least $1 \%$ completeness (top-coded at 100). This truncation or topcoding was used to avoid highly inflated estimates; Connecticut, North Carolina, and West Virginia were the jurisdictions affected. Even with weight truncation, estimates for Connecticut and North Carolina were highly unstable, resulting in inflated predicted estimates. These jurisdictions were therefore excluded from all analyses and, as a result, the findings of the analysis are not nationally representative.

\section{Validation of weighting strategies}

To evaluate the accuracy of the weighted predicted estimates, models were fit using data from May 10, 2020, to obtain predicted weekly counts of deaths from December 15, 2019, through April 5, 2020. These predicted counts were compared with the reported provisional counts available as of November 22, 2020, for the time period December 15, 2019, through April 5,2020 , approximately $6-11$ months following the week of death. While these reported provisional counts may still underestimate the eventual final count of deaths occurring in these several weeks, provisional data are generally more than $90 \%$ complete within 6 months of the date of death (5). Comparing the modelbased predicted counts to these reported provisional counts at longer lags can give an indication of which weighting scenario is likely to be more accurate. Comparisons were based on the mean percent difference between the modelbased (posterior medians) and reported provisional estimates, the RMSE, and trend plots of the reported and predicted counts.
Informed by the validation results, a fourth weighting scenario was constructed for the overall U.S. estimates based on the jurisdiction-specific improvements in timeliness. In this scenario, the predicted estimates for the United States were summed across the jurisdictions, where the timeliness scenario for each jurisdiction was chosen to minimize the RMSE. In other words, some jurisdictions may have demonstrated extreme improvements in timeliness, while others may have had median or moderate improvements.

\section{Trends}

To obtain values for the United States overall, posterior predicted estimates were summed over all of the jurisdictions, excluding Connecticut and North Carolina. Posterior predicted estimates (median and 95\% BCI) of the weekly number of deaths were obtained from the models and plotted to examine trends under the different timeliness scenarios. Trends were plotted using local regression (LOESS) smoothed values (16) with the ggplot2 package in R. Trends in the predicted numbers of deaths under different timeliness scenarios were plotted along with trends in the reported numbers of deaths after a 6- to 11-month lag to determine which scenario(s) most closely aligned with the trends in the reported number of deaths. These trend plots were visually inspected to determine which timeliness scenario(s) may better capture the direction and magnitude of trends in the numbers of deaths.

Based on the validation results, hierarchical Bayesian models were fit using data from 2016 through October 24,2020 , and the posterior predicted weekly estimates were plotted to examine trends in each of the outcomes. Additionally, trends in the predicted weekly numbers of deaths in 2020 were plotted relative to the average numbers of deaths in the corresponding weeks from 2016 through 2019 to highlight differences in 2020 relative to past years.

R statistical software (version 3.6.1; The R Foundation) was used to conduct

U.S. Department of Health and Human Services $•$ Centers for Disease Control and Prevention • National Center for Health Statistics • National Vital Statistics System 
all analyses. Hierarchical Bayesian models were fit using R-INLA, which relies on Integrated Nested Laplace Approximation to approximate the posterior marginals of latent Gaussian models $(17,18)$. More detail about the hierarchical Bayesian models can be found in the Technical Notes.

\section{Results}

\section{Validation}

\section{Drug overdose}

The results of the validation of the weighting strategies indicated that the scenario assuming that the timeliness of provisional data in 2020 was the same as the median levels in 2019 (first set of weights) resulted in predicted counts of deaths that were approximately $10.9 \%$ too high, on average, relative to the provisional counts observed on November 22, 2020. The scenario assuming that timeliness had improved moderately since 2019 (second set of weights) resulted in predicted counts that were $0.3 \%$ too high, on average.
The scenario assuming the largest improvements in timeliness resulted in predicted counts that were $9.5 \%$ too low, on average. The scenario allowing timeliness improvements to vary by jurisdiction resulted in predicted counts that were $4.0 \%$ too low, on average, but resulted in less week-toweek variability in the accuracy of the predicted counts (Table) with a smaller RMSE. Figure 1 shows the trends under different timeliness scenarios; the scenario allowing jurisdiction-specific improvements in timeliness most closely mirrored the trends in the reported counts. This is consistent with the results in the Table.

\section{Suicide}

The results of the validation of the weighting strategies indicated that the scenario assuming that the timeliness of provisional data in 2020 was the same as the median levels in 2019 (first set of weights) resulted in predicted counts of deaths that were approximately $2.1 \%$ too high, relative to the provisional counts observed on November 22, 2020. The scenario assuming that timeliness had improved moderately since 2019 (second set of weights) resulted in predicted counts that were $1.1 \%$ too low and had the lowest RMSE. The scenario assuming the largest improvements in timeliness resulted in predicted counts that were $4.8 \%$ too low. The scenario where timeliness improvements varied by jurisdiction resulted in predicted counts that were $1.8 \%$ too high, on average, and had the second lowest RMSE (Table). Figure 1 shows the trends under different timeliness scenarios. The scenario allowing jurisdictionspecific improvements in timeliness was similar to the scenario assuming timeliness was the same as median levels in 2019; both were slightly higher than the trends assuming moderate improvements in timeliness, which tended to underestimate the counts in the most recent 8 weeks.

\section{Transportation-related deaths}

The results of the validation of the weighting strategies indicated that the scenario assuming that the timeliness of provisional data in 2020 was the same as the median levels in 2019 (first set of

Table. Percent difference between reported weekly provisional counts of deaths (as of November 22, 2020) and initial predicted provisional counts (as of May 10, 2020) for drug overdose deaths, suicide, and transportation-related deaths, United States

Percent difference between predicted and observed provisional counts by timeliness scenario

\begin{tabular}{|c|c|c|c|c|c|}
\hline Lag (weeks) & $\begin{array}{l}\text { Week-ending } \\
\text { date of death }\end{array}$ & $\begin{array}{c}\text { Median values for } \\
2019\end{array}$ & $\begin{array}{c}\text { Moderate } \\
\text { improvement }\end{array}$ & $\begin{array}{c}\text { Extreme } \\
\text { improvement }\end{array}$ & $\begin{array}{l}\text { Jurisdiction-specific } \\
\text { improvement }\end{array}$ \\
\hline & & \multicolumn{4}{|c|}{ Drug overdose } \\
\hline 20 & $12 / 15 / 2019$ & -4.3 & -6.0 & -7.5 & -6.5 \\
\hline 19 & $12 / 22 / 2019$ & -2.8 & -4.8 & -6.8 & -5.5 \\
\hline 18 & $12 / 29 / 2019$ & -2.9 & -5.8 & -7.8 & -6.6 \\
\hline 17 & $1 / 5 / 2020$ & -3.3 & -6.1 & -8.4 & -6.8 \\
\hline 16 & $1 / 12 / 2020$ & -2.0 & -5.5 & -8.7 & -6.8 \\
\hline 15 & $1 / 19 / 2020$ & -1.0 & -5.4 & -9.5 & -7.1 \\
\hline 14 & $1 / 26 / 2020$ & 0.1 & -4.3 & -9.3 & -6.1 \\
\hline 13 & $2 / 2 / 2020$ & 4.5 & -0.9 & -7.5 & -3.7 \\
\hline 12 & $2 / 9 / 2020$ & 3.6 & -3.9 & -11.8 & -7.3 \\
\hline 11 & $2 / 16 / 2020$ & 5.6 & -1.3 & -9.0 & -4.5 \\
\hline 10 & $2 / 23 / 2020$ & 10.6 & 1.8 & -6.7 & -1.8 \\
\hline 9 & $3 / 1 / 2020$ & 11.7 & 0.7 & -9.7 & -3.9 \\
\hline 8 & $3 / 8 / 2020$ & 15.3 & -0.1 & -13.9 & -5.7 \\
\hline 7 & $3 / 15 / 2020$ & 11.6 & -4.0 & -18.9 & -10.9 \\
\hline 6 & $3 / 22 / 2020$ & 30.3 & 7.9 & -12.5 & -2.0 \\
\hline 5 & $3 / 29 / 2020$ & 45.8 & 16.8 & -8.5 & 5.0 \\
\hline 4 & $4 / 5 / 2020$ & 62.8 & 26.6 & -4.8 & 12.1 \\
\hline Overall & $\begin{array}{l}\text { Average percent } \\
\text { difference }\end{array}$ & 10.9 & 0.3 & -9.5 & -4.0 \\
\hline Overall & RMSE & 329 & 134 & 147 & 97 \\
\hline
\end{tabular}


Table. Percent difference between reported weekly provisional counts of deaths (as of November 22, 2020) and initial predicted provisional counts (as of May 10, 2020) for drug overdose deaths, suicide, and transportation-related deaths, United States-Con.

\begin{tabular}{|c|c|c|c|c|c|}
\hline \multirow[b]{2}{*}{ Lag (weeks) } & \multirow[b]{2}{*}{$\begin{array}{l}\text { Week-ending } \\
\text { date of death }\end{array}$} & \multicolumn{4}{|c|}{$\begin{array}{l}\text { Percent difference between predicted and } \\
\text { observed provisional counts by timeliness scenario }\end{array}$} \\
\hline & & $\begin{array}{l}\text { Median values for } \\
\qquad 2019\end{array}$ & $\begin{array}{c}\text { Moderate } \\
\text { improvement }\end{array}$ & $\begin{array}{c}\text { Extreme } \\
\text { improvement }\end{array}$ & $\begin{array}{l}\text { Jurisdiction-specific } \\
\text { improvement }\end{array}$ \\
\hline & & \multicolumn{4}{|c|}{ Suicide } \\
\hline 20 & $12 / 15 / 2019$ & -2.8 & -3.6 & -4.0 & -2.9 \\
\hline 19 & $12 / 22 / 2019$ & 1.0 & 0.3 & -0.1 & 1.2 \\
\hline 18 & $12 / 29 / 2019$ & 1.4 & 0.6 & 0.3 & 1.7 \\
\hline 17 & $1 / 5 / 2020$ & -4.5 & -5.6 & -5.9 & -4.6 \\
\hline 16 & $1 / 12 / 2020$ & 0.2 & -1.2 & -2.2 & -0.2 \\
\hline 15 & $1 / 19 / 2020$ & -2.3 & -3.6 & -4.6 & -2.4 \\
\hline 14 & $1 / 26 / 2020$ & 1.8 & 0.3 & -1.4 & 1.4 \\
\hline 13 & $2 / 2 / 2020$ & 0.4 & -1.9 & -4.5 & 0.2 \\
\hline 12 & $2 / 9 / 2020$ & 4.6 & 1.5 & -2.1 & 4.2 \\
\hline 11 & $2 / 16 / 2020$ & 5.3 & 1.5 & -4.3 & 5.3 \\
\hline 10 & $2 / 23 / 2020$ & 10.7 & 8.0 & -0.5 & 10.3 \\
\hline 9 & $3 / 1 / 2020$ & 11.1 & 6.9 & -1.0 & 10.6 \\
\hline 8 & $3 / 8 / 2020$ & -1.7 & -5.8 & -10.4 & -2.2 \\
\hline 7 & $3 / 15 / 2020$ & -1.8 & -6.6 & -11.4 & -2.0 \\
\hline 6 & $3 / 22 / 2020$ & 1.4 & -4.2 & -9.9 & 0.6 \\
\hline 5 & $3 / 29 / 2020$ & 5.9 & -2.0 & -8.9 & 5.1 \\
\hline 4 & $4 / 5 / 2020$ & 5.8 & -3.4 & -11.5 & 5.0 \\
\hline Overall & $\begin{array}{l}\text { Average percent } \\
\text { difference }\end{array}$ & 2.1 & -1.1 & -4.8 & 1.8 \\
\hline \multirow[t]{2}{*}{ Overall } & RMSE & 41 & 36 & 54 & 39 \\
\hline & & \multicolumn{4}{|c|}{ Transportation } \\
\hline 20 & $12 / 15 / 2019$ & 0.3 & -0.2 & -0.2 & 0.1 \\
\hline 19 & $12 / 22 / 2019$ & -0.3 & -1.2 & -1.5 & -0.5 \\
\hline 18 & $12 / 29 / 2019$ & -1.5 & -2.7 & -3.2 & -1.5 \\
\hline 17 & $1 / 5 / 2020$ & -2.2 & -3.1 & -3.4 & -2.3 \\
\hline 16 & $1 / 12 / 2020$ & 0.5 & 0.1 & -0.4 & 0.5 \\
\hline 15 & $1 / 19 / 2020$ & -1.1 & -2.3 & -3.4 & -0.4 \\
\hline 14 & $1 / 26 / 2020$ & 1.0 & -0.5 & -1.7 & 1.6 \\
\hline 13 & $2 / 2 / 2020$ & 3.1 & 1.4 & -0.6 & 3.3 \\
\hline 12 & $2 / 9 / 2020$ & 0.7 & -2.3 & -5.4 & 0.4 \\
\hline 11 & $2 / 16 / 2020$ & 6.6 & 3.7 & -0.3 & 6.4 \\
\hline 10 & $2 / 23 / 2020$ & 4.2 & 0.9 & -4.7 & 3.8 \\
\hline 9 & $3 / 1 / 2020$ & 5.1 & 1.7 & -3.7 & 4.6 \\
\hline 8 & $3 / 8 / 2020$ & -0.9 & -4.4 & -8.9 & -1.0 \\
\hline 7 & $3 / 15 / 2020$ & -0.2 & -4.2 & -9.1 & -0.4 \\
\hline 6 & $3 / 22 / 2020$ & 0.8 & -3.5 & -8.4 & 0.1 \\
\hline 5 & $3 / 29 / 2020$ & 1.2 & -4.7 & -11.4 & 0.2 \\
\hline 4 & $4 / 5 / 2020$ & 12.2 & 3.7 & -5.0 & 11.3 \\
\hline Overall & $\begin{array}{l}\text { Average percent } \\
\text { difference }\end{array}$ & 1.7 & -1.0 & -4.2 & 1.4 \\
\hline Overall & RMSE & 24 & 19 & 36 & 22 \\
\hline
\end{tabular}

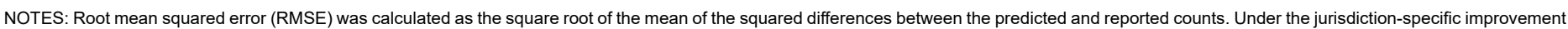

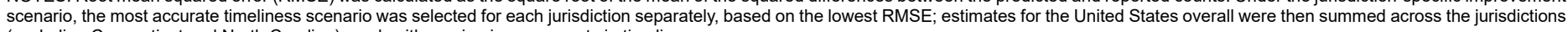
(excluding Connecticut and North Carolina), each with varying improvements in timeliness.

SOURCE: National Center for Health Statistics, National Vital Statistics System, December 2019 to April 2020.

weights) resulted in predicted counts of deaths that were approximately $1.7 \%$ too high, relative to the provisional counts observed on November 22, 2020. The scenario assuming that timeliness had improved moderately since 2019 (second set of weights) resulted in predicted counts that were $1.0 \%$ too low and had the lowest RMSE. The scenario assuming the largest improvements in timeliness resulted in predicted counts that were $4.2 \%$ too low. The scenario where timeliness improvements varied by jurisdiction resulted in predicted counts that were $1.4 \%$ too high, on average, with the second lowest RMSE (Table). Figure 1 shows the trends under different timeliness scenarios; the scenario allowing jurisdiction-specific improvements in timeliness was similar to the scenario assuming timeliness 
Figure 1. Comparison of reported and predicted counts of drug overdose, suicide, and transportation-related deaths under different timeliness scenarios

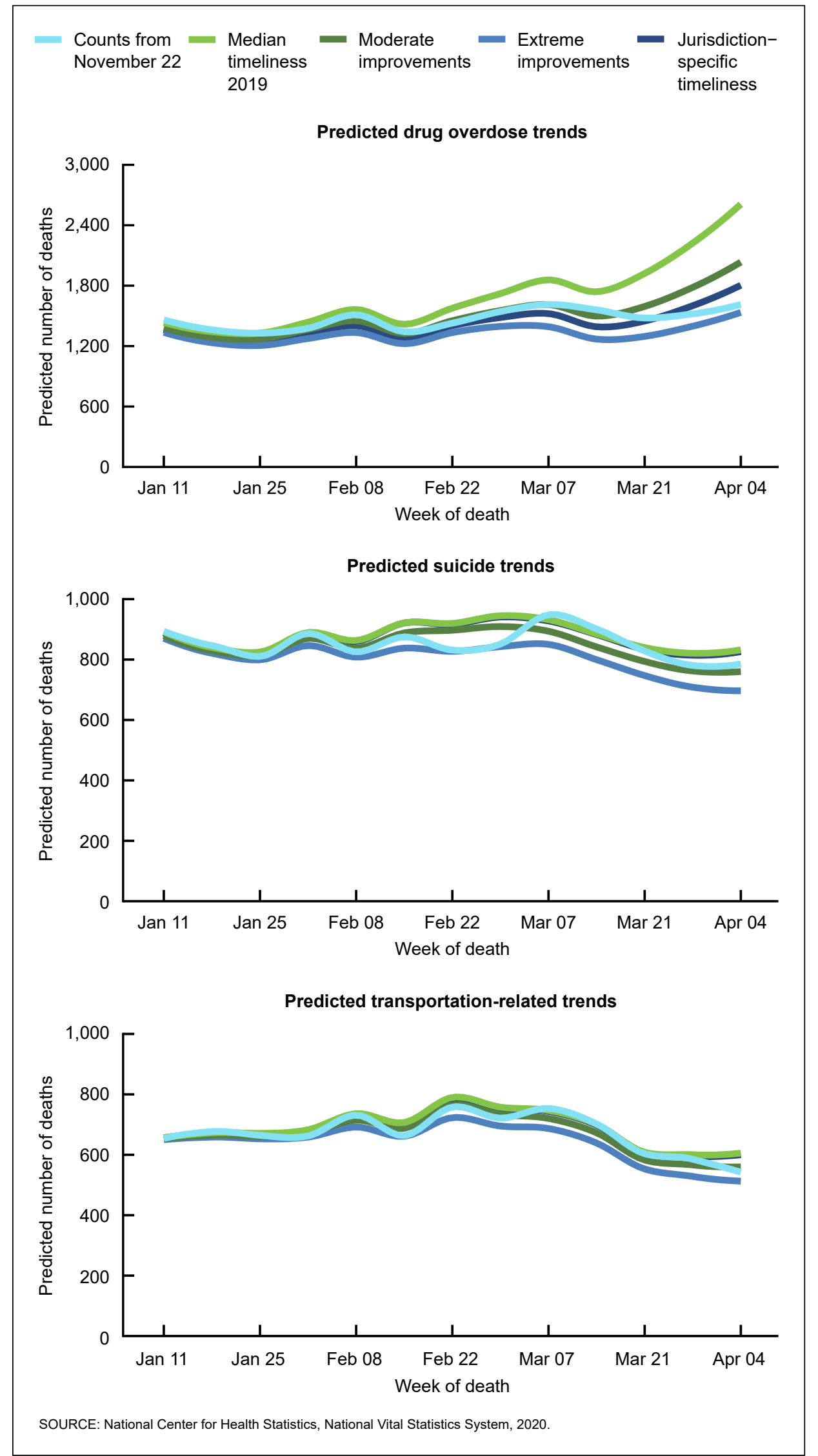

was the same as median levels in 2019; both were slightly higher than the trends assuming moderate improvements in timeliness, which tended to underestimate the counts in the most recent 8 weeks.

Overall, the validation suggested moderate improvement in timeliness for drug overdose deaths, but smaller improvements in timeliness for suicide and transportation-related deaths in early 2020. Less variability was observed between the various timeliness scenarios for suicide and transportationrelated deaths than was observed for drug overdose deaths, particularly at shorter lags. The trends based on jurisdiction-specific improvements in timeliness more closely mirrored the observed counts for drug overdose deaths and had the lowest RMSE. For suicide and transportation-related deaths, estimates based on jurisdictionspecific improvements in timeliness or moderate improvements in timeliness resulted in similar RMSEs and average percent differences, with the moderate improvements in timeliness scenario slightly outperforming the jurisdictionspecific timeliness scenario. However, accounting for jurisdiction-specific improvements in timeliness appeared to better capture the more recent temporal trends (Figure 1). Thus, for consistency, trends in the predicted numbers of deaths reported below are based on the estimates allowing jurisdiction-specific improvements in timeliness. Supplemental Figures I-III (see Technical Notes) show the predicted estimates under different timeliness scenarios by jurisdiction and outcome relative to the observed counts from November 22, 2020.

\section{Trends}

Based on the validation, hierarchical Bayesian models assuming jurisdictionspecific improvements in timeliness were fit for each outcome, and the posterior predicted number of deaths from 2016 through October 24, 2020, were plotted to examine trends over the past several years. Additionally, trends 
in the predicted weekly numbers of deaths in 2020 were plotted relative to the average numbers of deaths in the corresponding weeks from 2016 through 2019 to highlight differences in 2020 relative to past years.

\section{Drug overdose}

Looking at the trends in the predicted weekly numbers of drug overdose deaths since 2016, the predicted counts have been increasing since early 2019. These increases appear to have accelerated since February 2020 and may have declined in more recent months (Figure 2). Figure 3 displays the weekly predicted counts in 2020, assuming jurisdiction-specific improvements in timeliness since 2019, relative to the average counts in the same weeks of 2016-2019 combined.

\section{Suicide}

Looking at the trends in the weekly numbers of suicide deaths since 2016 , strong seasonal patterns were evident with the numbers highest in the middle of the year and lowest in winter months (Figure 4). Figure 5 displays the weekly predicted counts in 2020, assuming jurisdiction-specific improvements in timeliness, relative to the average counts in the same weeks of 2016-2019 combined. Predicted weekly numbers of suicide deaths were similar to historic levels in early 2020, then declined from March through June, and remain slightly lower than historic levels in more recent months.

\section{Transportation}

Looking at the trends in the weekly numbers of transportation-related deaths since 2016, strong seasonal patterns were evident with the numbers highest in the middle of the year and lowest in winter months (Figure 6). Figure 7 displays the weekly predicted counts in 2020, assuming jurisdiction-specific improvements in timeliness, relative to the average counts in the same weeks of 2016-2019 combined. Predicted weekly numbers of transportation-related deaths were similar to historic levels in early 2020, then declined from March through April, and increased in more recent months.

Jurisdiction-specific trends can be seen in Supplemental Figures IV-VI (see Technical Notes).

\section{Discussion}

This report develops and describes nowcasting methods to estimate recent trends in deaths due to external causes, accounting for reporting delays and potential improvements in timeliness since 2019. The accuracy of estimated trends in external causes of death is dependent on how timeliness has changed over time, including since the COVID-19 pandemic. The validation analysis suggests that timeliness has improved since 2019, with larger improvements for drug overdose deaths than for suicide and transportationrelated deaths. For all outcomes, the

Figure 2. Trends in predicted weekly numbers of drug overdose deaths: United States, 2016-October 24, 2020

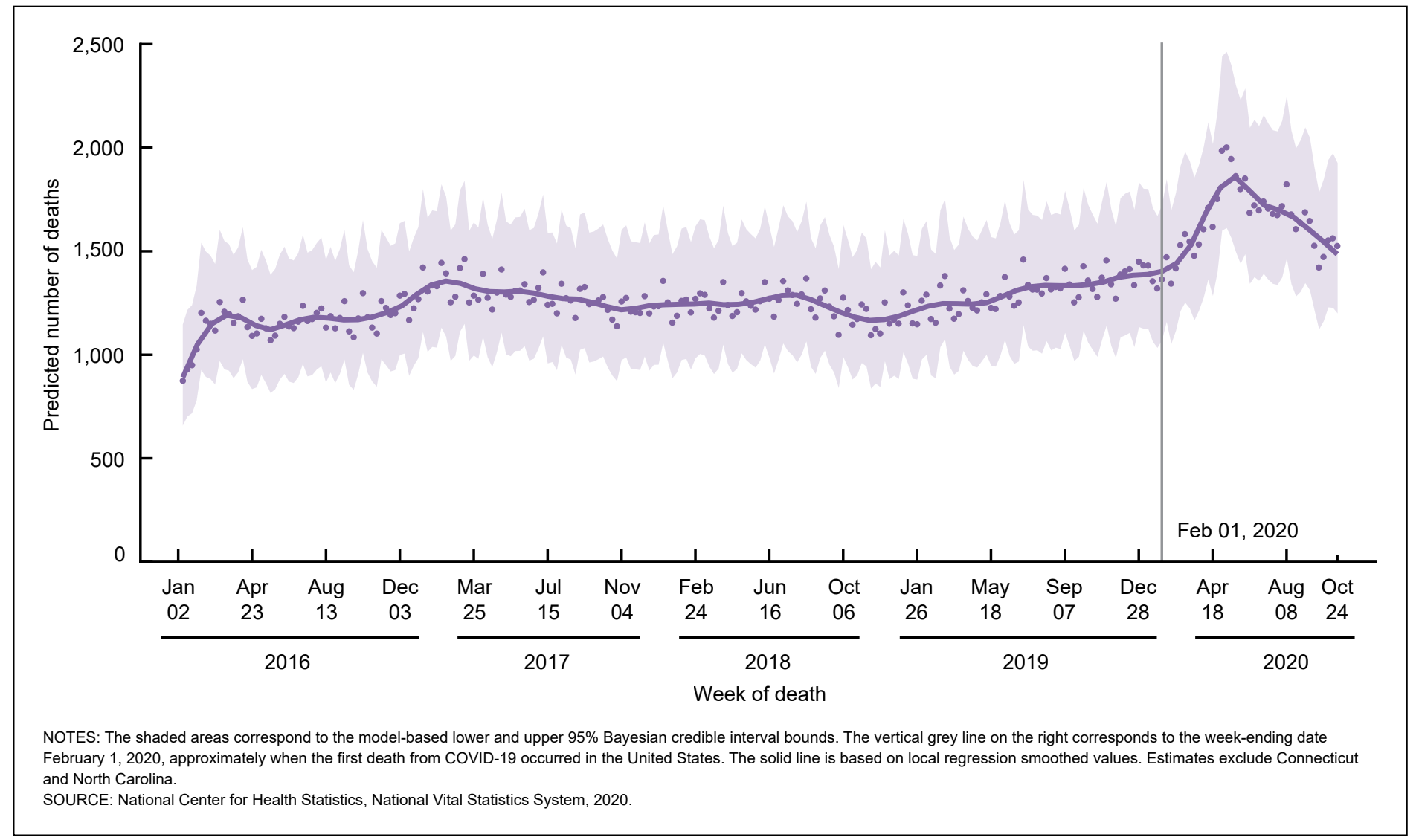

U.S. Department of Health and Human Services $\bullet$ Centers for Disease Control and Prevention • National Center for Health Statistics $\bullet$ National Vital Statistics System 


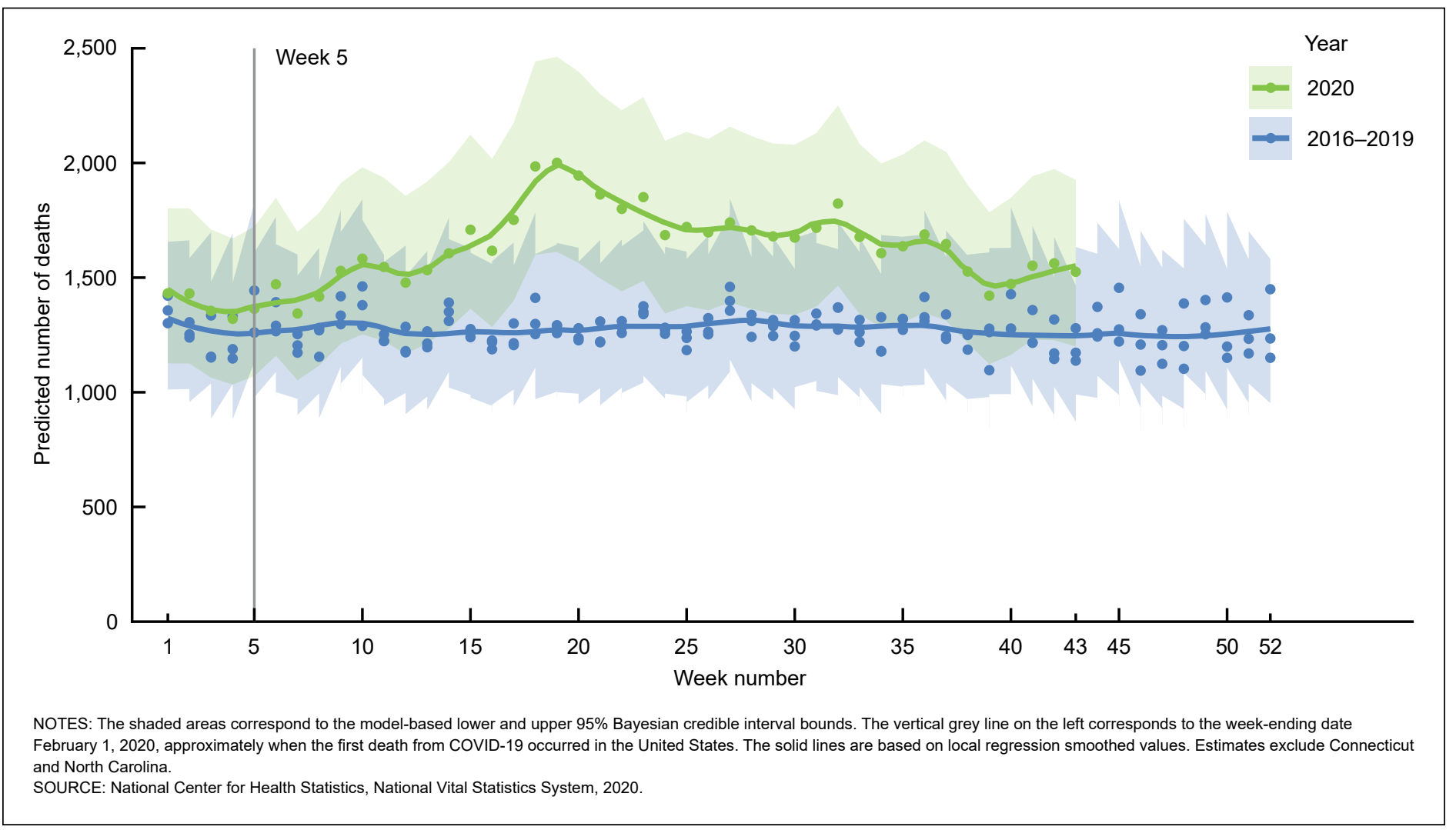

Figure 4. Trends in predicted weekly numbers of suicide deaths: United States, 2016-October 24, 2020

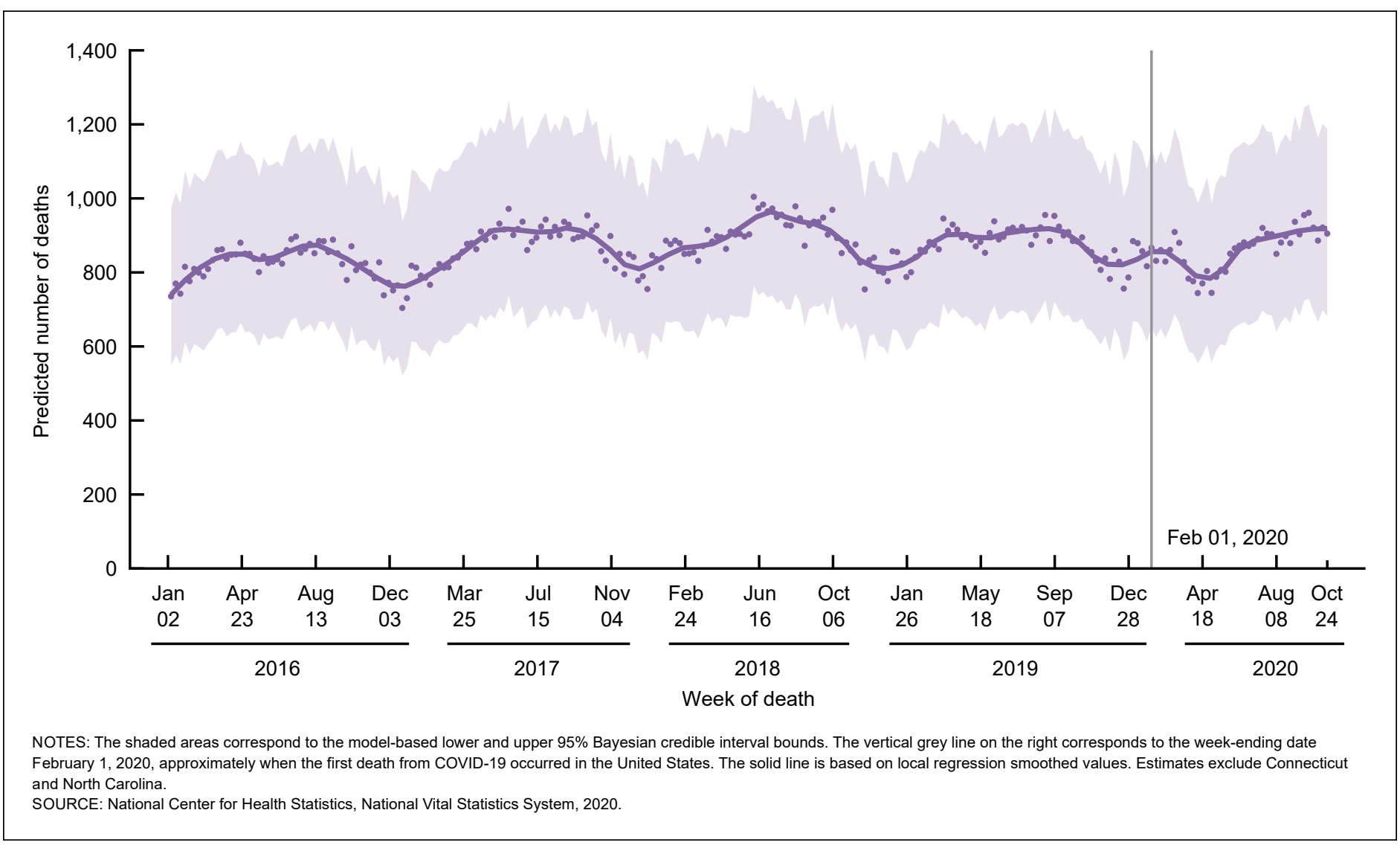

U.S. Department of Health and Human Services • Centers for Disease Control and Prevention • National Center for Health Statistics $\bullet$ National Vital Statistics System 


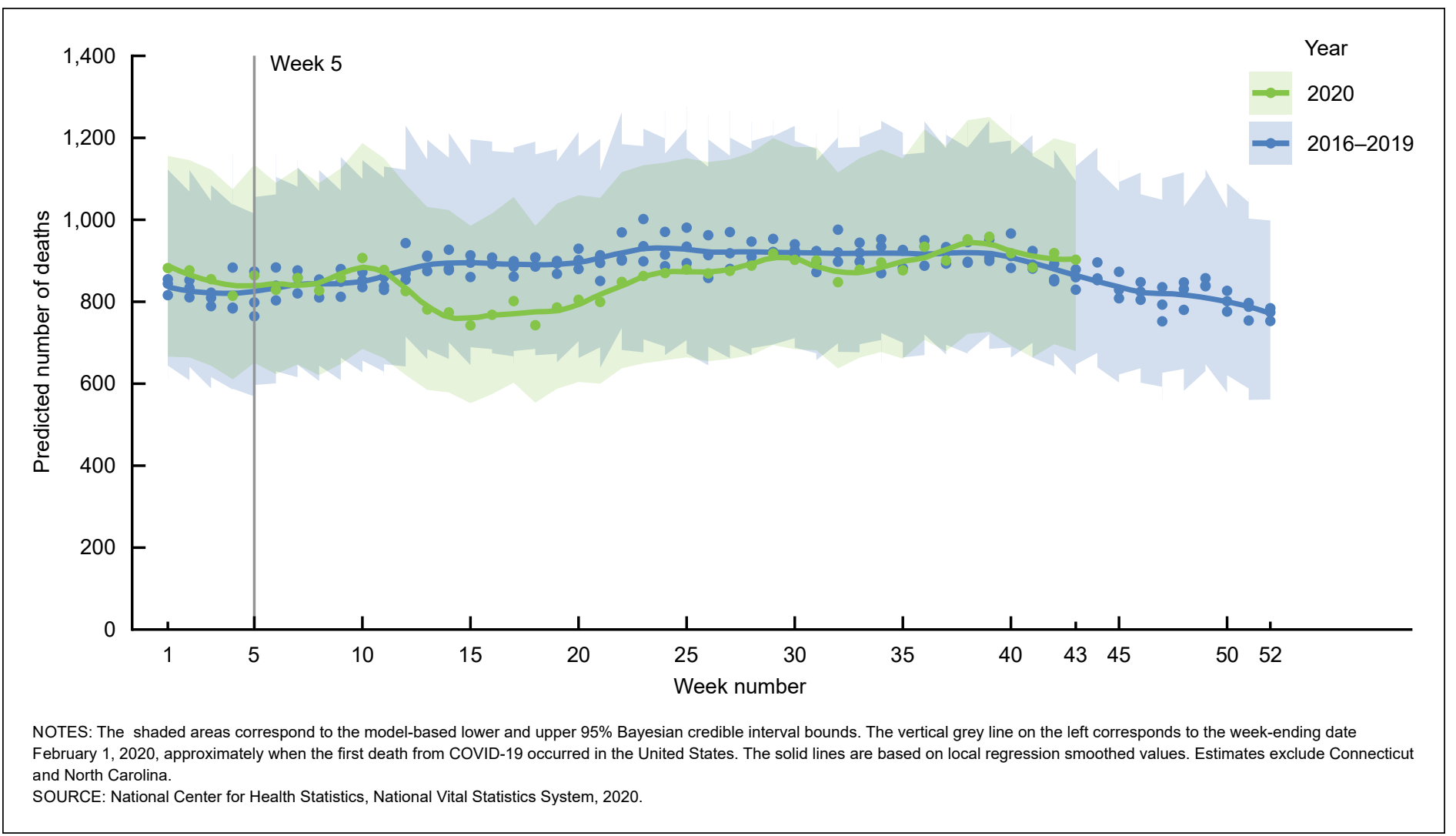

Figure 6. Trends in predicted weekly numbers of transportation-related deaths: United States, 2016-October 24, 2020

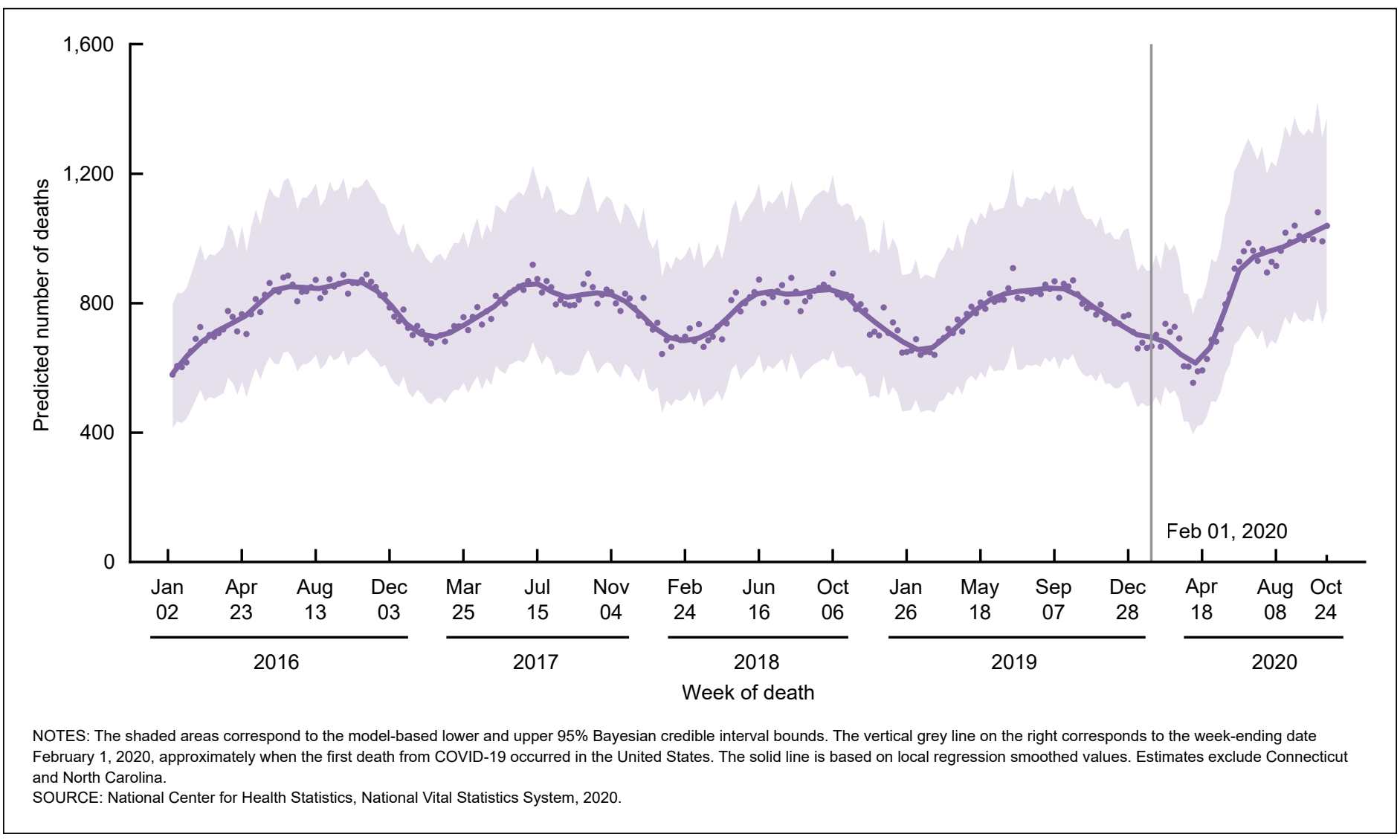

U.S. Department of Health and Human Services $\bullet$ Centers for Disease Control and Prevention $\bullet$ National Center for Health Statistics $\bullet$ National Vital Statistics System 
Figure 7. Weekly predicted counts of transportation-related deaths: United States, 2016-2019 and 2020

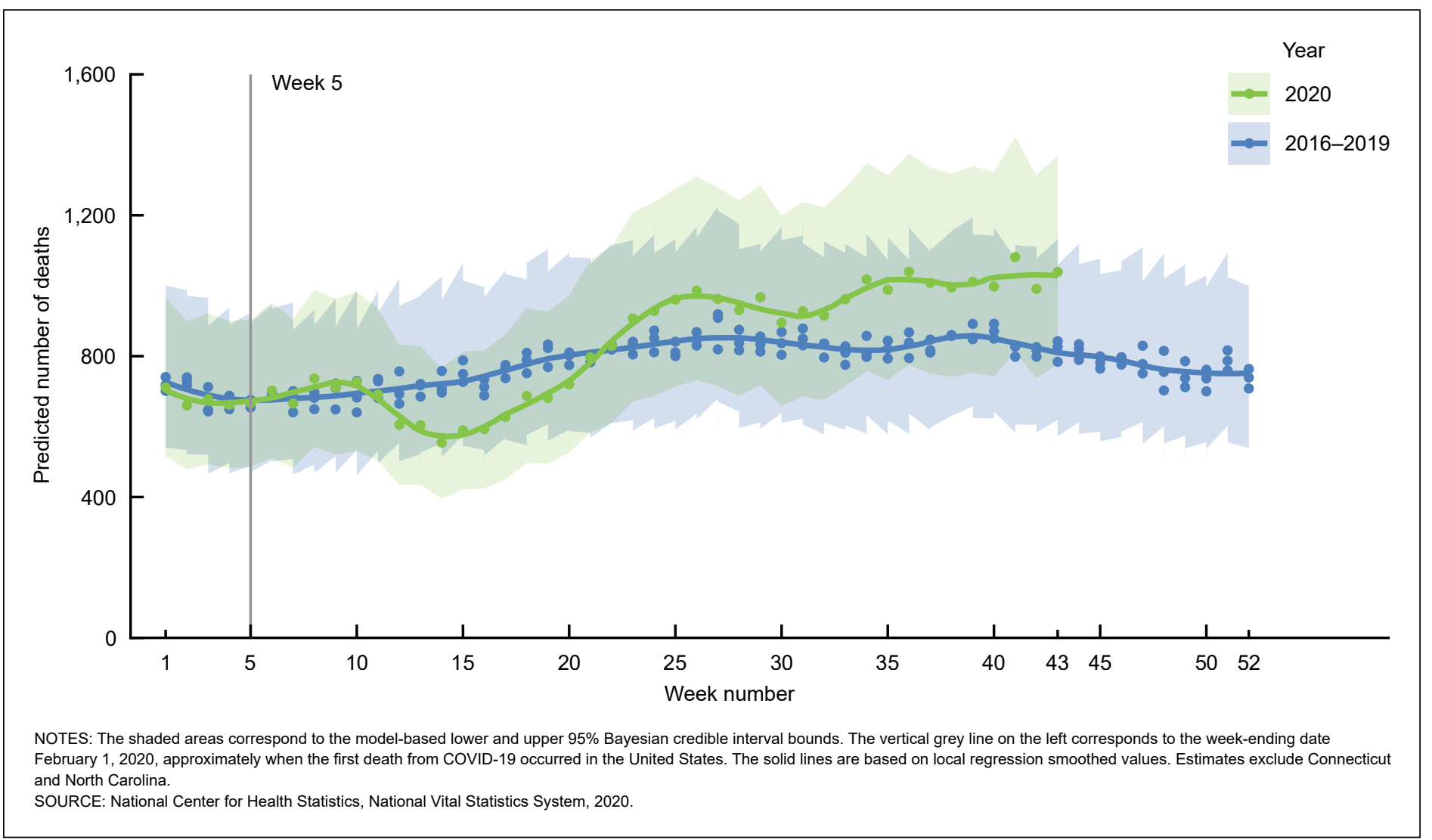

scenario assuming that timeliness was similar in 2020 to median levels in 2019 resulted in predicted estimates that were too large, on average, relative to the reported counts $6-11$ months later, when counts should be nearly complete. Accounting for jurisdictionspecific improvements in timeliness, predicted provisional counts as of May 10,2020 , were, on average, within $4.0 \%$, $1.8 \%$, and $1.4 \%$ of eventual reported counts for drug overdose, suicide, and transportation-related deaths, respectively. However, it is possible that provisional data for those weeks are still incomplete, and that the degree of difference will change as more data are received. There may have also been improvements in timeliness since April or May 2020, which would lead to the weighted estimates being too high in recent months. There have been substantial investments in improving the timeliness of drug overdose death data over the past few years $(4,7)$, and it is unclear how the pace of these improvements may have changed since the beginning of the pandemic. Although little evidence supports this to date, decreases in timeliness may have occurred in some jurisdictions. For example, timeliness may have been affected in jurisdictions with large numbers of deaths from COVID-19, which created additional burdens on local death investigation and registration systems.

Results of this analysis suggest that after accounting for reporting delays and improvements in timeliness in 2020 relative to 2019, weekly numbers of drug overdose deaths have increased since early 2019 through May 2020, with the rate of increase potentially accelerating from February to May 2020. Weekly counts may have declined since May, though there is a greater amount of uncertainty associated with recent estimates both because the provisional data are less complete, and it is unknown how timeliness may have changed in more recent months. Trends in suicide deaths appear to have potentially declined or remained stable relative to past years. Trends in transportationrelated deaths, the majority of which are motor vehicle traffic deaths (94\%), initially declined in March but then increased from April through June. Importantly, the magnitude and direction of these recent trend estimates depend on how timeliness has changed in recent months. For example, if timeliness has improved to a larger extent in recent months, the recent trends will be overestimated. If timeliness has worsened, then recent trends may be underestimated. The degree to which timeliness may have changed throughout 2020 will not be known until data are finalized in late 2021.

The findings from the nowcasting estimates mirror observational information in recent reports. For example, the American Medical Association published an issue brief highlighting reports of increases in opioid-related overdose during the COVID-19 pandemic, including reports 
from individual states (19). A study from Kentucky reported an increase in emergency medical services encounters for opioid overdose prior to and after stay-at-home orders were placed in Kentucky for COVID-19 (20). A study of data from Massachusetts found no increases in suicide deaths from March 2020 through May 2020 (21). A report from the National Safety Council based on data from all 50 states indicated that the fatality rate per miles driven in May 2020 , when much of the country was in lockdown from the pandemic, increased $23.5 \%$ compared with the previous year, despite a $25.5 \%$ decrease in vehicle miles driven compared with the year prior. Overall, the mileage death rate per 100 million vehicle miles driven was 1.47 in May 2020 compared with 1.19 in 2019 (22).

This analysis has several limitations. First, the timeliness of provisional death certificate data varies by jurisdiction and over time. Many jurisdictions responded to the pandemic by submitting data more frequently or expanding their use of electronic death registration systems. Conversely, data for a few jurisdictions may have been more delayed due to staffing challenges, data processing disruptions, coding delays, and other factors. Recent changes in timeliness will likely not be apparent until provisional data are more complete, typically 6 to 9 months or more after the date of death. Second, it is possible that improvements to timeliness have occurred at shorter lags, but not necessarily at longer lags. Thus, the scenarios examined may not necessarily be accurate if improvements in timeliness have occurred at shorter lags only. The scenarios explored in this report assumed fixed levels of improvement across time. Third, the hierarchical Bayesian models smooth extreme values by borrowing strength over neighboring states and over time. Thus, the predicted values may shrink extreme counts, especially where data are sparse. When evaluating the impact of the pandemic on these outcomes, estimates may be affected by this smoothing or shrinkage. Fourth, estimates for the United States overall do not include data from Connecticut and North Carolina. With additional improvements in timeliness, it is possible that provisional data for Connecticut and North Carolina may be available for inclusion in the future. Finally, while the initial predicted counts from certain models were within, on average, $5 \%$ of the eventual reported counts for the United States overall, there is likely a much wider degree of variability across jurisdictions. Ongoing evaluations of the timeliness and accuracy of predicted counts by jurisdiction will help to inform future adjustments to the nowcasting methods and facilitate a better understanding of the accuracy and uncertainty of the predicted provisional counts.

\section{Conclusions}

Applying nowcasting methods to estimate trends in drug overdose, suicide, and transportation-related deaths, accounting for reporting lags, allows for more timely publication of provisional death counts. These modelbased provisional estimates can be used to better understand the potential impact of the COVID-19 pandemic on injuryrelated causes of death under different scenarios about the change in timeliness. The accuracy of these provisional estimates depends on the assumptions made regarding how timeliness has changed since the beginning of the pandemic. While various models were fit to explore possible changes in timeliness, the true patterns in reporting lags will not be known until data are finalized, typically $11-12$ months following the end of the data year.

\section{References}

1. Murphy SL, Xu JQ, Kochanek KD, Arias E, Tejada-Vera B. Deaths: Final data for 2018. National Vital Statistics Reports; vol 69 no 13. Hyattsville, MD: National Center for Health Statistics. 2020. Available from: https://www.cdc.gov/nchs/ data/nvsr/nvsr69/nvsr69-13-508.pdf.
2. National Center for Health Statistics. National Vital Statistics System, Rapid Release Program. Quarterly provisional estimates for selected indicators of mortality, 2018-Quarter 2, 2020. Available from: https:// www.cdc.gov/nchs/nvss/vsrr/ mortality.htm.

3. Provisional drug overdose death counts. National Center for Health Statistics. National Vital Statistics System, Vital Statistics Rapid Release Program. 2020. Available from: https://www.cdc.gov/nchs/ nvss/vsrr/drug-overdose-data.htm.

4. Spencer MR, Ahmad F. Timeliness of death certificate data for mortality surveillance and provisional estimates. Vital Statistics Rapid Release; no 1. Hyattsville, MD: National Center for Health Statistics. January 2017. Available from: https://www.cdc.gov/nchs/data/vsrr/ report001.pdf.

5. Ahmad FB, Dokpesi P, Escobedo L, Rossen L. Timeliness of death certificate data by sex, age, and geography. Vital Statistics Rapid Release; no 9. Hyattsville, MD: National Center for Health Statistics. June 2020. Available from: https:// www.cdc.gov/nchs/data/vsrr/ vsrr009-508.pdf.

6. Rossen LM, Ahmad FB, Spencer MR, Warner M, Sutton P. Methods to adjust provisional counts of drug overdose deaths for underreporting. Vital Statistics Rapid Release; no 6. Hyattsville, MD: National Center for Health Statistics. August 2018. Available from: https://www.cdc. gov/nchs/data/vsrr/report006.pdf.

7. Ahmad FB, Spencer MR. Timeliness of death certificate data for suicides. Vital Statistics Rapid Release; no 3. Hyattsville, MD: National Center for Health Statistics. April 2018. Available from: https://www.cdc. gov/nchs/data/vsrr/report003.pdf.

8. Rossen LM, Womack LS, Spencer MR, Ahmad FB. Timeliness of infant death data for infant mortality surveillance and quarterly 
provisional estimates. Vital Statistics Rapid Release; no 5. Hyattsville, MD: National Center for Health Statistics. June 2018. Available from: https://www.cdc.gov/nchs/data/vsrr/ report005.pdf.

9. National Center for Health Statistics. National Vital Statistics System, Rapid Release Program. COVID-19 death data and resources. Available from: https://www.cdc.gov/nchs/ nvss/covid-19.htm.

10. Green HK, Andrews NJ, Bickler G, Pebody RG. Rapid estimation of excess mortality: Nowcasting during the heatwave alert in England and Wales in June 2011. J Epidemiol Community Health 66:866-8. 2012.

11. Nunes B, Natario I, Carvalho ML. Nowcasting influenza epidemics using non-homogeneous hidden Markov models. Statist Med 32:2643-60. 2013.

12. Bastos LS, Economou T, Gomes MFC, Villeia DA, Coelho FC, Cruz $\mathrm{OG}$, et al. A modelling approach for correcting reporting delays in disease surveillance data. Stat Med 38(22):4363-77. 2019.

13. Besag J, York J, Mollié A. Bayesian image restoration, with two applications in spatial statistics. Ann Instit Stat Math 43(1):1-20. 1991.

14. Watanabe S. A widely applicable Bayesian information criterion. J Mach Learn Res 14:867-97. 2013.

15. Lee DT, Schacter BJ. Two algorithms for constructing a Delaunay triangulation. Int J Computer Information Sciences 9(3):219-42. 1980.

16. Cleveland WS, Grosse E, Shyu WM. Chapter 8. Local regression models. In: Chambers SJM and Hastie T, editors. Statistical models in S. Chapman \& Hall/CRC. 309-73. 1992.

17. Khan D, Rossen LM, Hedegaard $\mathrm{H}$, Warner M. A Bayesian spatial and temporal modeling approach to mapping geographic variation in mortality rates for subnational areas with R-INLA. J Data Sci 16(1):147-82. 2018.

18. Rue H, Martino S, Chopin N. Approximate Bayesian inference for latent gaussian models by using integrated nested laplace approximations. J R Stat Soc Series B 71(2):319-92. 2009.

19. American Medical Association. Issue brief: Reports of increases in opioidand other drug-related overdose and other concerns during COVID pandemic. 2020. Available from: https://www.ama-assn.org/system/ files/2020-09/issue-brief-increasesin-opioid-related-overdose.pdf.

20. Slavova S, Rock P, Bush HM, Quesinberry D, Walsh SL. Signal of increased opioid overdose during COVID-19 from emergency medical services data. Drug Alcohol Depend 214(9):108176. 2020.

21. Faust JS, Shah SB, Du C, Li SX, Lin Z, Krumholz HM. Suicide deaths during the stay-at-home advisory in Massachusetts. medRxiv 2020. Available from: https://www. medrxiv.org/content/10.1101/2020.1 $0.20 .20215343 \mathrm{v} 1$.

22. National Safety Council. Motor vehicle fatality rates rose $23.5 \%$ in May, despite quarantines. 2020. Available from: https://www.nsc. org/in-the-newsroom/motor-vehiclefatality-rates-rose-235-in-maydespite-quarantines. 


\section{Technical Notes}

\section{Hierarchical spatial model specification}

All hierarchical Bayesian models were fit via Integrated Nested Laplace Approximation (INLA) in R (https://www.r-inla.org/).

\section{Estimating completeness of provisional data}

Completeness of provisional counts, $y_{t, d, s}$, relative to final counts, $n_{t, ., s}$ (which do not vary by delay), was estimated using zero-inflated binomial models, for $s=1, \ldots, S$ jurisdictions and $t=1, \ldots, T$ time (weeks), and $d=1, \ldots, D$ delay (weeks):

$$
\begin{gathered}
y_{t, d, s} \sim\left\{\begin{array}{cc}
0 & \text { with probability } p_{t, d, s} \\
\operatorname{Binomial}\left(n_{t, ., s}, \lambda_{t, d, s}\right) & \text { with probability } 1-p_{t, d, s}
\end{array}\right. \\
\operatorname{logit}\left(\lambda_{t, d, s}\right)=\mu+\alpha_{t}+\beta_{d}+\psi_{s}+\gamma_{d, s}+\delta_{t, s}
\end{gathered}
$$

This model includes:

a) A logit link function $\log \left(\lambda_{t, d, s} /\left(1-\lambda_{t, d, s}\right)\right)$; where $\lambda_{t, d, s}$ is the proportion of deaths reported (provisional count divided by the final count) at time $t$, with delay $d$, in jurisdiction $s$.

b) An overall intercept term $\mu$, assigned a flat prior.

c) A random effect for time, $\alpha_{t}$, which smooths estimates over time using a first order random walk (where values for a given time, $t$, depend on values in the prior time, $t-1)$.

d) A random effect for the delay, $\beta_{d}$ (first order random walk).

e) A random effect for jurisdiction, $\psi$, which accounts for jurisdiction-level variation.

f) A jurisdiction-delay interaction term, $\gamma_{d, s}$, which accounts for any residual variation in the delay by jurisdiction.

g) A jurisdiction-time interaction term, $\delta_{t, s}$, which accounts for any residual variation over time, by jurisdiction.

These last three random effects were assumed to be independently and identically distributed, and the conditional precisions of the random effects are assigned logGamma (1, 0.001) priors (by default in R-INLA) $(17,18)$.

Alternative models included binomial models, which resulted in poorer model fit, based on the Watanabe-Akaike Information Criterion (WAIC) (14).

\section{Estimating provisional counts of death}

Provisional counts of death, $y_{t, s}$, were estimated using zero-inflated Poisson models, for $s=1, \ldots, S$ jurisdictions and $t=1, \ldots, T$ time (weeks):

$$
\begin{gathered}
y_{t, s} \sim\left\{\begin{array}{cl}
0 & \text { with probability } p_{t, s} \\
\operatorname{Poisson}\left(\lambda_{t, s}\right) & \text { with probability } 1-p_{t, s}
\end{array}\right. \\
\log \left(\lambda_{t, s}\right)=\mu+\alpha_{t}+u_{s}+v_{s}+\delta_{t, s}
\end{gathered}
$$

This model includes:

a) A log link function $\log \left(\lambda_{t, s}\right)$; where $\lambda_{t, s}$ is the mean number of deaths reported at time $t$, in jurisdiction $s$. b) An overall intercept term, $\mu$, assigned a flat prior.

c) A random effect for time, $\alpha_{t}$, which smooths estimates over time using a first order random walk (where values for a given time, $t$, depend on values in the prior time, $t-1)$.

d) A Besag-York-Mollié spatial random effect, $u_{s}$, to account for spatial dependence (e.g., clustering) across jurisdictions (13). This term was modeled using conditionally autoregressive (CAR) priors where weights were assigned to each jurisdiction according to adjacency; neighboring jurisdictions receive a weight of 1 , while non-neighboring jurisdictions receive a weight of 0 . This term also includes a nonspatial random effect, $v_{s}$, to account for residual jurisdictionlevel variation that is not spatially dependent.

Jurisdictions were considered neighbors based on Delaunay triangulation (15), which generates Voronoi triangles from jurisdiction centroids, where nodes connected by a triangle edge are considered neighbors. This spatial weighting scheme ensures that each area has at least one neighbor.

e) A jurisdiction-time interaction term, $\delta_{t, s}$, which accounts for any residual variation over time, by jurisdiction, assumed to be independently and identically distributed, and the conditional precisions of the random effects are assigned log-Gamma $(1,0.001)$ priors (by default in R-INLA) (17,18).

Alternative models included Poisson models, which resulted in poorer model fit, based on the WAIC (14). 
Figure I. Predicted weekly estimates of drug overdose death counts in early 2020 under different timeliness scenarios, compared with reported counts, by jurisdiction

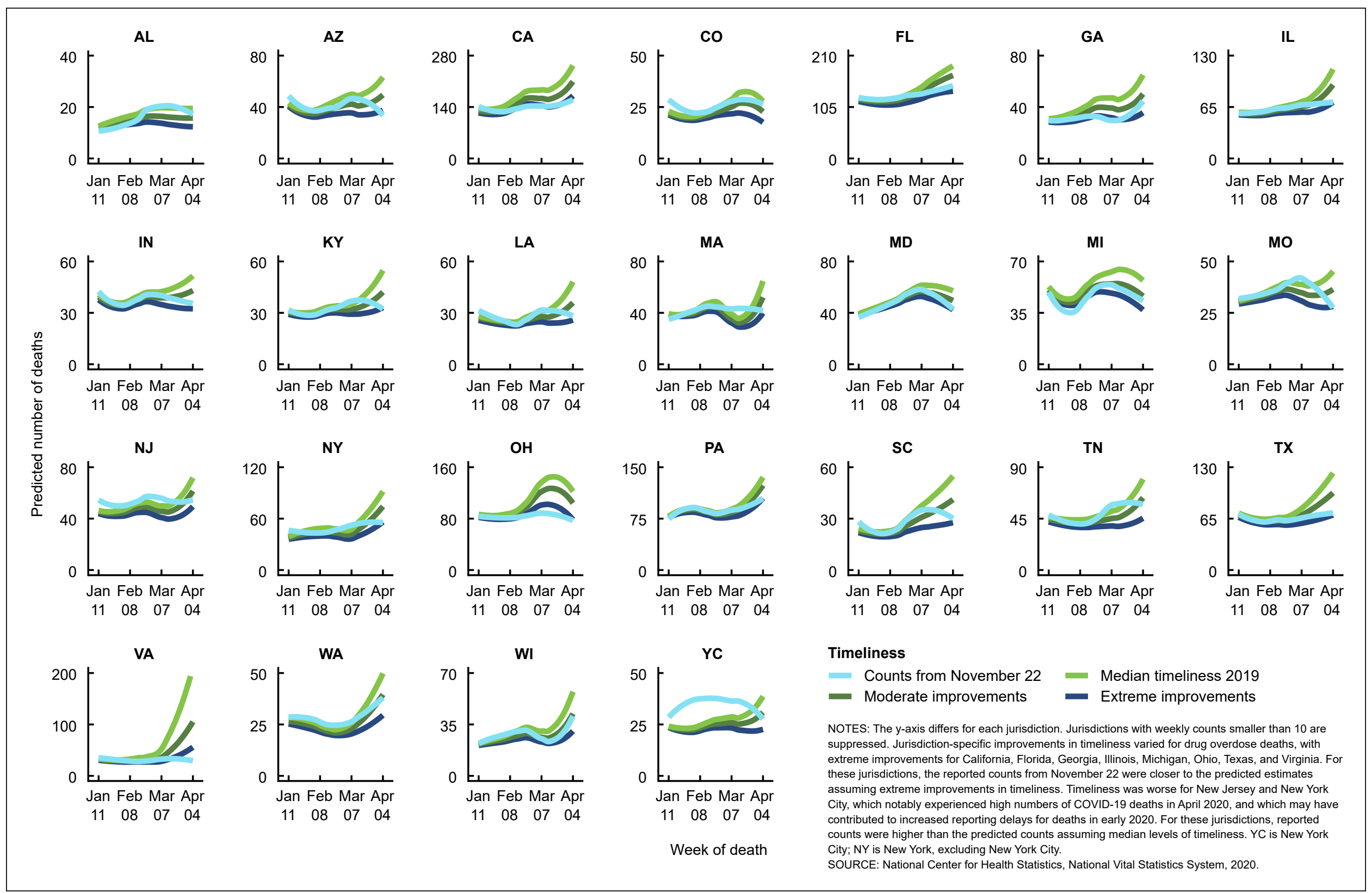




\section{Vital Statistics Surveillance Report}

Figure II. Predicted weekly estimates of suicide death counts in early 2020 under different timeliness scenarios, compared with reported counts, by jurisdiction

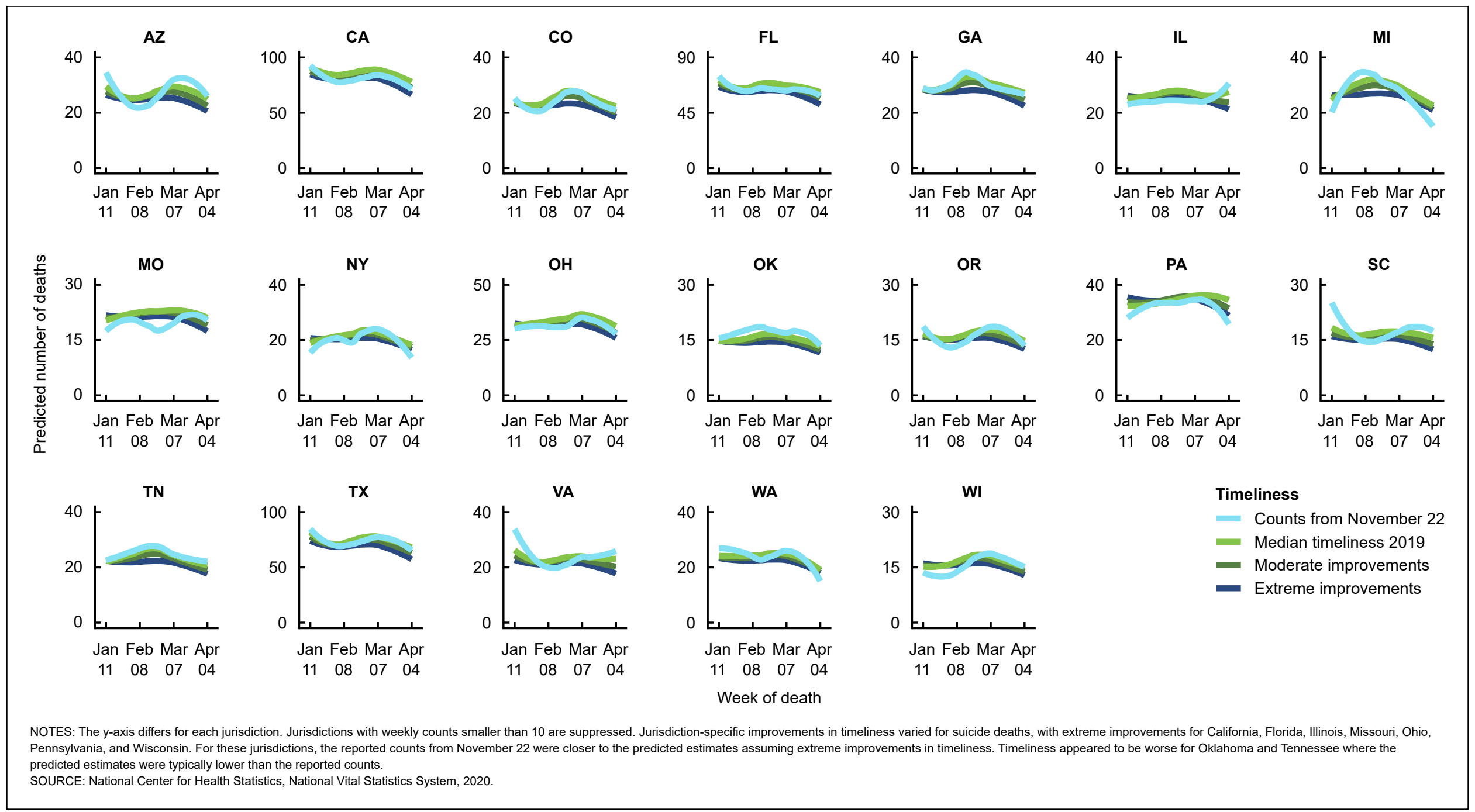




\section{Vital Statistics Surveillance Report}

Figure III. Predicted weekly estimates of transportation-related death counts in early 2020 under different timeliness scenarios, compared with reported counts, by jurisdiction

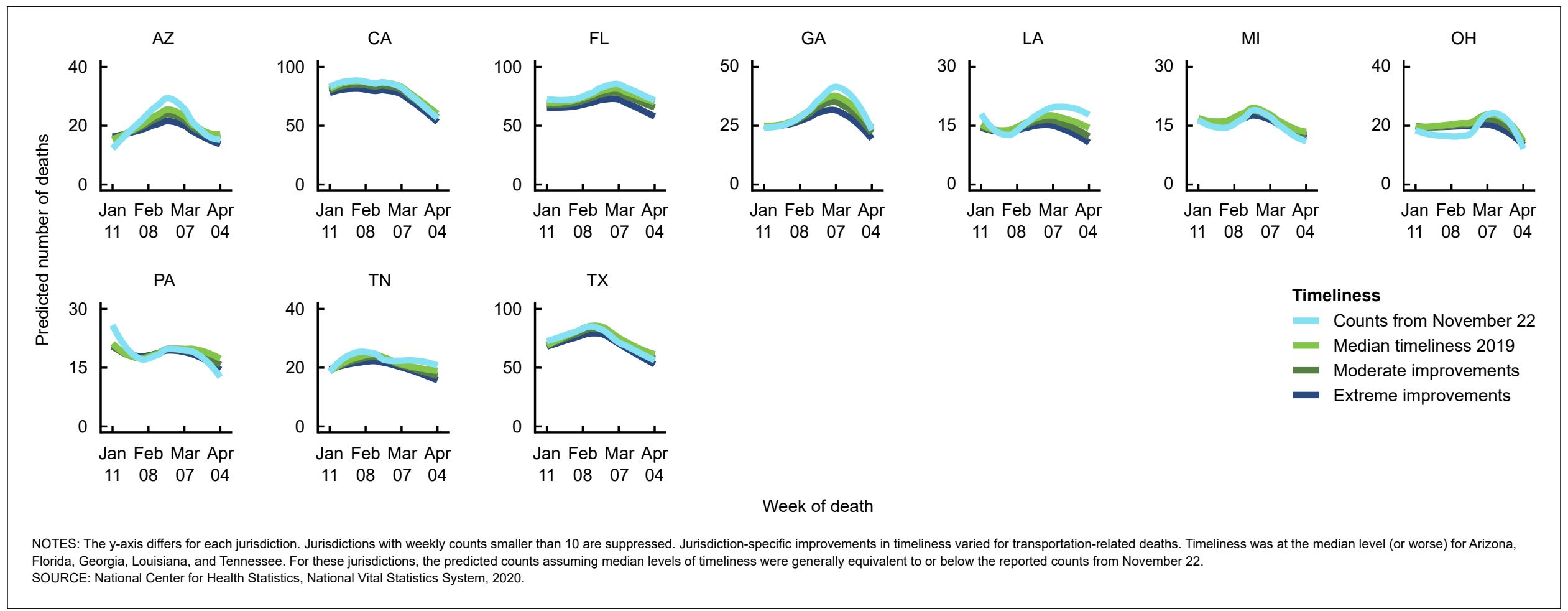




\section{Vital Statistics Surveillance Report}

Figure IV. Predicted weekly counts of drug overdose deaths in 2020 relative to the corresponding weeks of 2016-2019, by jurisdiction

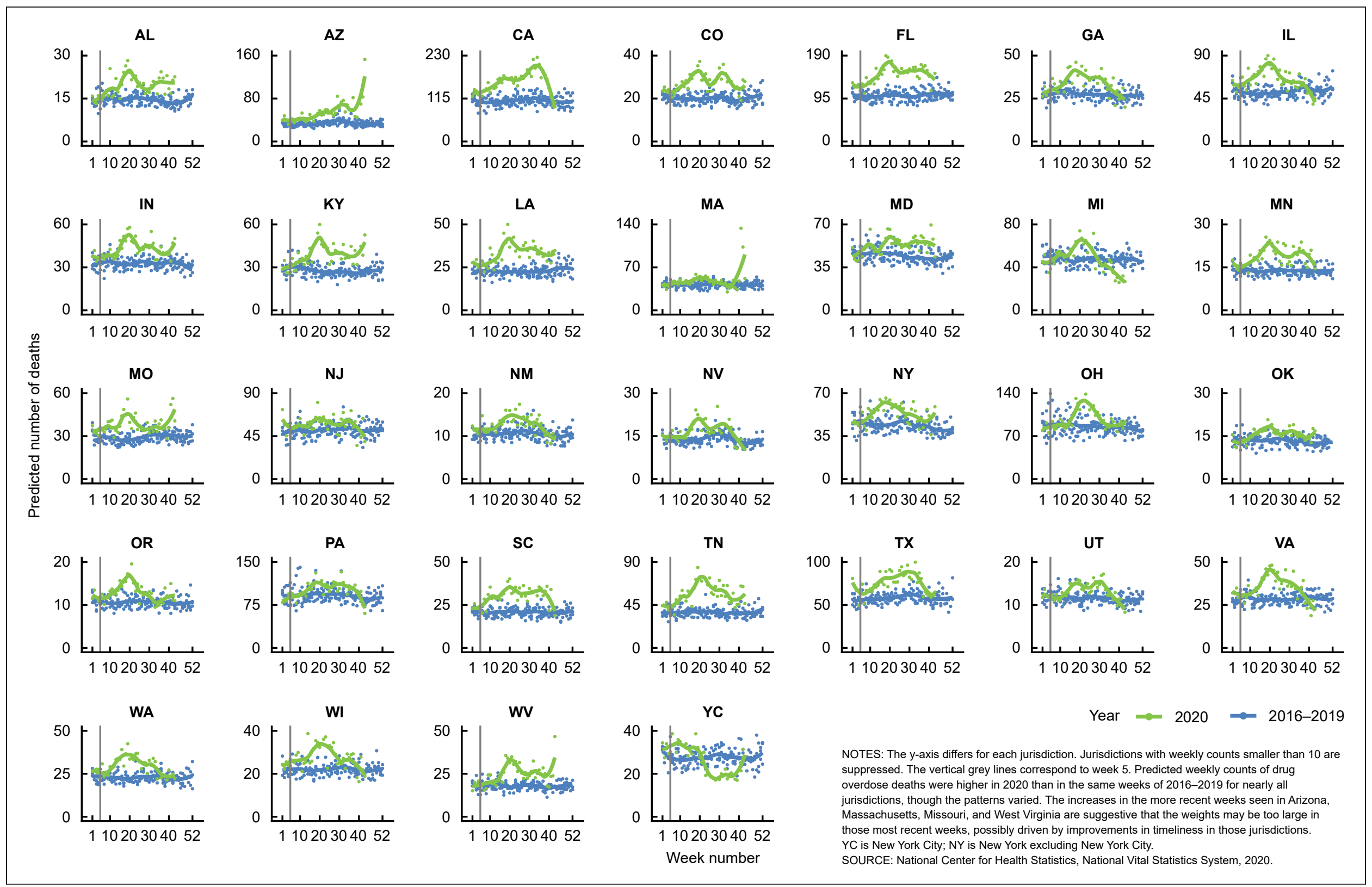


Figure V. Predicted weekly counts of suicide deaths in 2020 relative to the corresponding weeks of 2016-2019, by jurisdiction

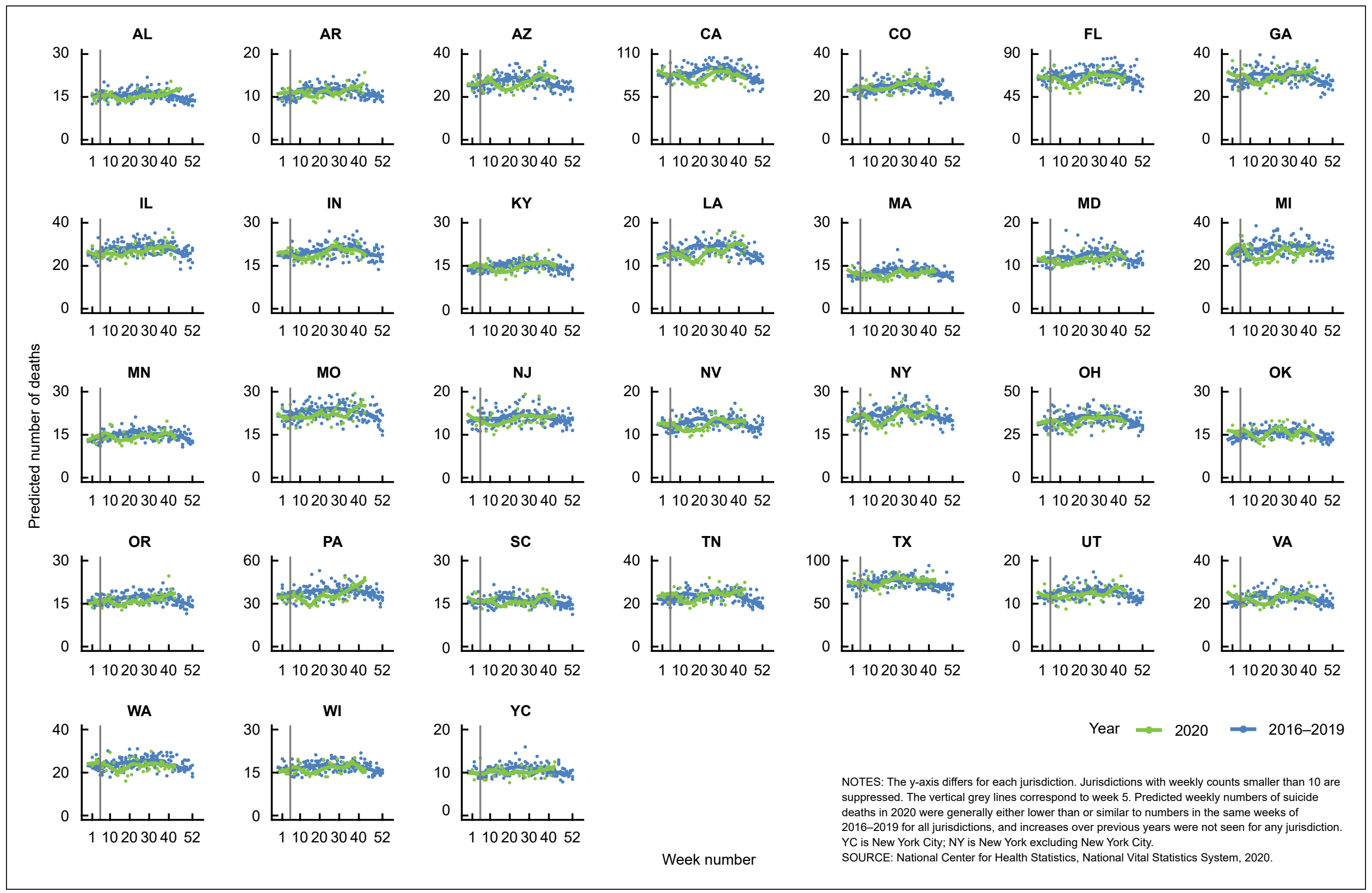




\section{Vital Statistics Surveillance Report}

Figure VI. Predicted weekly counts of transportation-related deaths in 2020 relative to the corresponding weeks of 2016-2019, by jurisdiction

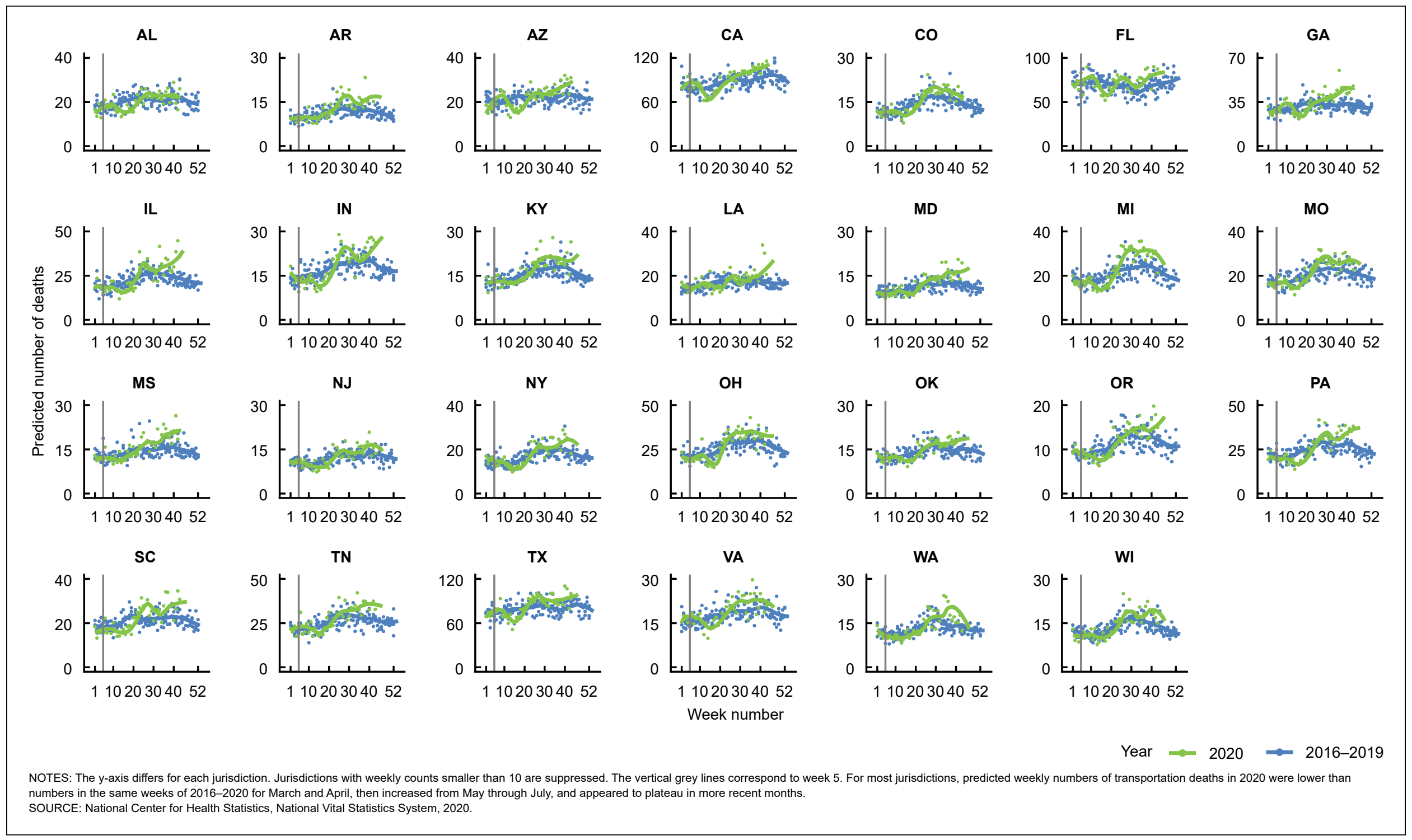




\section{Suggested citation}

Rossen LM, Hedegaard H, Warner M, Ahmad FB, Sutton PD. Early provisional estimates of drug overdose, suicide, and transportation-related deaths: Nowcasting methods to account for reporting lags. Vital Statistics Rapid Release; no 11. Hyattsville, MD: National Center for Health Statistics. February 2021. DOI: https://doi.org/10.15620/ cdc:101132.

\section{Copyright information}

All material appearing in this report is in the public domain and may be reproduced or copied without permission; citation as to source, however, is appreciated.

\section{National Center for Health Statistics}

Brian C. Moyer, Ph.D., Director

Amy M. Branum, Ph.D., Acting Associate Director for Science

Division of Research and Methodology

Jennifer D. Parker, Ph.D, Director

John Pleis, Ph.D., Acting Associate

Director for Science

Division of Analysis and Epidemiology

Irma E. Arispe, Ph.D, Director

Kevin Heslin, Ph.D., Associate Director for Science

\section{Division of Vital Statistics}

Steven Schwartz, Ph.D, Director

Isabelle Horon, Dr.P.H., Acting Associate

Director for Science 